\title{
EDUCACIÓN, ARTE Y NATURALEZA EN WILLIAM T. HARRIS Y MANUEL B. COSSÍO
}

\author{
Education, art and nature in William T. Harris and Manuel B. Cossío
}

\section{Eugenio Otero Urtaza*}

Fecha de recepción: 11/07/2016 • Fecha de aceptación: 23/10/2016

Resumen. William Torrey Harris (1835-1909) es un educador de gran importancia en los Estados Unidos: es el eslabón que enlaza el pensamiento de Horace Mann con John Dewey. Harris conoció a Giner y Cossío en París en 1889 y desde entonces mantuvo una relación estable con la Institución Libre de Enseñanza (ILE), que facilitó un intercambio de información y un mayor conocimiento en España de las ideas pedagógicas que se estaban desarrollando en su país. Se estudian así los primeros contactos entre la pedagogía norteamericana y la ILE, y se hace un recorrido por la biografía de Harris hasta que fue nombrado comisario de educación de los Estados Unidos, especialmente en lo que fue su primer contacto con el trascendentalismo y su conexión posterior con el pensamiento de Hegel, de su preocupación por integrar todo ese pensamiento en la idea del kindergarten como un espacio netamente público, de convivencia entre todos los grupos y clases sociales. En la centralidad del estudio se examinan algunas concomitancias y diferencias entre el pensamiento de Harris y el de Cossío en relación con la capacidad educadora del arte y la naturaleza, especialmente en el ámbito escolar y la formación técnica, concluyéndose que ambos forman parte de una red trasnacional que impregna el pensamiento pedagógico más allá de los marcos nacionales que Kloppenberg denomina «transatlantic community of discourse», un complejo proceso de convergencia intelectual que buscaba una vía intermedia entre el idealismo y el positivismo.

Palabras clave: William T. Harris; Manuel B. Cossío; Francisco Giner de los Ríos; Institución Libre de Enseñanza; Trascendentalismo norteamericano; Educación artística; Educación y naturaleza.

Abstract. William Torrey Harris (1835-1909) was an educator of great importance in the United States and can be seen as the link between the thought of

\footnotetext{
" Departamento de Pedagogía y Didáctica. Facultad de Formación del Profesorado. Universidad de Santiago de Compostela (Campus de Lugo). Avenida de Ramón Ferreiro s/n, 27002 Lugo. España. otero.urtaza@usc.es.
}

Cómo citar este artículo: Otero Urtaza, Eugenio. «Educación, arte y naturaleza en William T. Harris y Manuel B. Cossío», Historia y Memoria de la Educación, 5 (2017): 15-71. 
Horace Mann and John Dewey. Harris met Giner and Cossio in Paris in 1889 and from that point on maintained a stable relationship with the Institucion Libre de Enseñanza (Free Institution of Teaching, ILE). This allowed for an exchange of information and increased the knowledge in Spain of the pedagogical ideas that were being developed in his country. This paper looks at the first contacts between American pedagogy and the ILE, before walking us through Harris' biography until he was appointed commissioner of education in the United States. We focus on his initial contact with transcendentalism and its subsequent connection with the thought of Hegel and on his wish to integrate all of these concepts into the idea of kindergarten as a fundamentally public space of coexistence for people of all groups and social classes. The core of the study offers a review of some similarities and differences between the thought of Harris and Cossio in connection with the educational potential of art and nature, especially regarding schools and technical training, concluding that both are part of a transnational trend in pedagogical thinking that goes beyond national frameworks. This "transatlantic community of discourse», as Kloppenberg called it, is a complex process of intellectual convergence that sought a middle way between idealism and positivism.

Keywords: William T. Harris; Manuel B. Cossio; Francisco Giner de los Ríos; Institución Libre de Enseñanza; American transcendentalism; Artistic education; Education and nature.

\section{INTRODUCCIÓN}

Las relaciones de la Institución Libre de Enseñanza (en adelante ILE) con los educadores de los Estados Unidos es un episodio que apenas ha sido tratado en lo que hace referencia a sus inicios en el siglo XIX, y sin embargo su presencia se hace sentir casi desde el mismo momento de su creación, cuando James Russell Lowell apareció por Madrid como jefe de la legación norteamericana, en lo que fue su primer cargo político. ${ }^{1}$ No cabe duda de que esta relación fue creciente en el primer tercio del siglo xx, y que, incluso terminada la Guerra Civil, hubo muchos intelectuales, científicos y escritores exiliados que encontraron acogida en sus universidades, en contraste vivo con los que fueron recibidos oficialmente después de 1953. No obstante, hay unos primeros anclajes pedagógicos

\footnotetext{
${ }^{1}$ Permaneció en Madrid entre 1877 y 1879 apoyando activa y continuadamente la labor de la ILE. Luego fue embajador en Londres donde volvió a encontrarse con Giner y Cossío en 1884. Sobre su estancia en España se publicó un libro póstumo: Impressions of Spain (Boston/New York, Houghton, Mifflin and Company, 1899). Para su relación con la ILE, véase Lawrence L. Klibbe, «James Russell Lowell's Residence in Spain», Hispania 41 (2), (1958): 190-194.
} 
que forjaron una conexión temprana que se fue agrandando con el tiempo y que permitieron la recepción de su cultura en España antes de 1936.

Muchos años antes de que llegasen allí los primeros pensionados, ya se hablaba en Estados Unidos de lo que significaba... la creación de Giner. En 1884, la publicación de una nota en el informe anual del Comisariado de Educación, descubre el interés que empezaba a suscitar la ILE en ese país. La nota menciona en tonos muy elogiosos sus ensayos para conformar el sistema de enseñanza a los principios de la pedagogía moderna, y destaca que los más eminentes hombres de ciencia, escritores, artistas y políticos del país le prestaban su apoyo con sus publicaciones, lo que estaba sirviendo para mejorar las deficiencias de la educación universitaria. Añadía que practicaban el excursionismo escolar como no se hacía entonces en ningún otro país de Europa, y que habían logrado que el francés fuese introducido en los planes de estudio oficiales y esperaban conseguir pronto que lo fuese la gimnasia. ${ }^{2}$

Es conocido que Giner y Cossío, al contrario que otros institucionistas, como Labra o Posada, no mostraron demasiado interés en conocer lo que se estaba realizando en las repúblicas hispanoamericanas, e incluso en la única oportunidad que tuvo Cossío de desplazarse a Argentina, renunció a hacerlo. ${ }^{3}$ Volcados en la proyección europea y con deseos de que España alcanzara la prosperidad de otras naciones del continente apenas dedicaron esfuerzos a conocer la realidad escolar del antiguo imperio colonial. Pero sí mostraron mucho interés por conocer la potencia industrial e intelectual en que se estaban convirtiendo los Estados Unidos, y pocos años después de terminada la contienda por la independencia de Cuba en 1898, Cossío cruzó el Atlántico en 1904 para participar en el Congreso de Artes y Ciencias, y en la Exposición Universal de Saint Louis, visitando también Chicago, Filadelfia y Nueva York donde tomó contacto con educadores por los que sentía cierta afinidad como William Wistar Comfort (1874-1955), Isaac Sharpless (1848-1920), John D. Fitz-Gerald (1873-1946) o Benjamín Richard Andrews (1877-1963). Un viaje que dio origen a unos incipientes contactos que se ampliarían en las primeras

\footnotetext{
${ }^{2}$ Report of the Commissioner of Education for the Year 1883-1884 (Washington: Government Printing Office, 1885), ccxxxii-xxxiv. Consultado en http://catalog.hathitrust.org/Record/009164574 (Cornell University).

${ }^{3}$ Eugenio Otero Urtaza: Manuel Bartolomé Cossío trayectoria vital de un educador (Madrid: Publicaciones de la Residencia de Estudiantes/CSIC, 1994), 344.
} 
décadas del siglo Xx, con la política de la Junta para Ampliación de Estudios y especialmente después de que Federico de Onís se instalase en la Universidad de Columbia.

Conviene subrayar así, que el interés por la cultura y la educación que surgía en los Estados Unidos es coetáneo al propio nacimiento de la ILE. En el curso 1878-1879, Rafael María de Labra había impartido un curso sobre sus instituciones políticas, y Adolfo A. Buylla publicó en 1886 un artículo sobre el desarrollo de las ideas socialistas en ese país, recordando que había sido un laboratorio de muchos experimentos sociales, deteniéndose en las huelgas y las luchas obreras que entonces se producían. Revisó la función que cumplía la emigración alemana, nutrida de numerosos refugiados que llegaron a partir de 1848, los Knights of Labour, la International Workingmen's Association y el Social Labour Party, y divulgando las ideas que Richard Ely había publicado en Recent American Socialism. ${ }^{4}$

Con todos estos antecedentes, Giner y Cossío conocieron a William Torrey Harris en París en 1889. ${ }^{5}$ Este encuentro marca el principio de una relación que se extiende hasta los primeros años del siglo $\mathrm{xx}$, mientras Harris fue Comisario del Bureau de Educación, y que tuvo como primer resultado que Cossío empezara a interesarse por la organización escolar que existía en los Estados Unidos, ${ }^{6}$ y que Harris enviase a España las publicaciones del Bureau y los informes del comisariado que en ocasiones comentaría Giner en el BILE. En ese encuentro de 1889 descubrieron que Harris tenía una manera de pensar que conectaba con los deseos de reforma social que la ILE estaba proponiendo a la sociedad española y, en 1891, Giner se hizo eco de sus convicciones sobre la educación moral y la necesidad de la separación de las confesiones religiosas

\footnotetext{
${ }^{4}$ Adolfo Álvarez Buylla, «El socialismo en los Estados Unidos», BILE, X (220), (1886): 98.

${ }^{5}$ En realidad, Harris y Cossío habían coincidido en el Congreso Internacional de Enseñanza de Bruselas, en 1880, que había sido organizado por la Ligue Belge de l'Enseignement, aunque no hay constancia que hubiesen mantenido entonces ninguna conversación.

${ }^{6}$ A finales de noviembre de 1889, Cossío publicaría en el BILE un artículo titulado «La enseñanza en los Estados Unidos y su organización». Casi todas las fuentes que utiliza provienen de Ferdinand Buisson, pero por primera vez utiliza el Report of the Commissioner, que posiblemente le había enviado Harris, que acaba de ocupar su puesto en el Bureau of Education de Washington. Al año siguiente publicó «Notas sobre la Inspección escolar en los Estados Unidos» con apoyo bibliográfico también de Buisson. Es posible que Buisson mediara en el encuentro de Harris con Cossío y Giner en 1889 .
} 
de las instituciones civiles, especialmente de la escuela primaria. ${ }^{7}$ Conforme a ideas que ya había expresado Horace Mann con anterioridad, y que compartían plenamente Giner y Cossío, creía que en la escuela solo podían explicarse principios que fuesen comunes a todos los credos y que, por otra parte, era necesario promover en ella el conocimiento y el amor hacia los grandes principios que rigen las relaciones ideales entre cada persona y sus semejantes. ${ }^{8}$

Es especialmente significativo que cuando se hundió el Maine y se inició la guerra de Cuba en 1898 fue el BILE, y posiblemente por iniciativa directa de Giner, la única revista que se atrevió en España a difundir elogiosamente las ideas educativas norteamericanas. Dio noticia de diversos «credos» que defendían sus grandes educadores como Francis Wayland Parker (1837-1902), James Laughlin Hughes (1846-1935), Richard. G. Boone (1849-1923), Lewis Henry Jones (1844-1917), Levi Seeley (1847-1928), Edward Wheeler Scripture (1864-1945), Earl Barnes (1861-1935), Burke Aaron Hinsdale (1837-1900), Reginald Heber Holbrook (1845-1910), y por supuesto John Dewey, con quien se explaya más que con ningún otro. Sigue con John S. Clark, profesor de arte en Boston, que comenta a Dewey, y Patterson du Bois (1847-1917), para terminar con el análisis de las ideas de Harris. ${ }^{9}$ No es cuestión de comenzar haciendo un análisis pormenorizado de la pedagogía de este último, pero conviene traer a la actualidad la importancia que concedía en su credo a la naturaleza como primer educador, aunque aseveraba que era luego en las instituciones donde el individuo realmente cogía fuerza: «diez robinsones obrando juntos - decía- no son iguales a diez individuos, sino a diez veces diez». Para él lo «esencial es la

\footnotetext{
${ }^{7}$ Francisco Giner de los Ríos, «La moral en la escuela según el Dr. Harris», BILE, XV (345), (1891): 184-7.

${ }^{8}$ Neil Gerard Mccluskey, Public Schools and Moral Education: The influence of Horace Mann, William Torrey Harris, and John Dewey (New York: Columbia University Press, 1958), 145.

${ }^{9}$ Los «credos» de estos educadores, firmados por X, un pseudónimo que solía utilizar Giner, aparecieron en el BILE en cinco entregas durante ese año de 1889, y habían sido publicados previamente en The School Journal, revista editada en Nueva York y Chicago. Sobre esta cuestión, véase Rosa Bruno-Jofré y Gonzalo Jover Olmeda, «El ideal democrático en el ideario pedagógico americano de finales del siglo XIX y su transposición en dos escenarios de habla hispana», en El largo camino hacia una educación inclusiva: la educación especial y social del siglo XIX a nuestros días: XV Coloquio de Historia de la Educación, Pamplona-Iruñea, 29, 30 de junio y 1 de julio de 2009, Vol. 1, coords. María Reyes Berruezo Albéniz y Susana Conejero López (Pamplona: Universidad Pública de Navarra, 2009), 23-34. Como señalan Bruno-Jofré y Jover, Dewey apenas era conocido entonces en España y efectivamente la presentación biográfica que se hace de Harris es mucho más amplia. No obstante, el texto recogido de Dewey es el más extenso de todos.
} 
acción del todo social» si bien consideraba que no se podían descuidar las peculiaridades del individuo «y a contentarse con asegurar que aprenda ese saber consolidado y a subordinarse a las maneras y costumbres de los demás hombres: hacerle "obediente"; y no solo a las leyes del Estado, sino hasta a las reglas convencionales de la etiqueta; sobre todo a sus padres, a sus maestros y a sus mayores». ${ }^{10}$

Harris es un pensador muy relevante, con raíces en el pensamiento europeo e interesado en crear redes educativas trasnacionales. Fue un importante referente de la educación norteamericana para los institucionistas, y por ello merece ser mejor conocido en España. Si Giner y Cossío se interesaron por Harris es realmente porque vieron que su pensamiento fortalecía la pedagogía que ellos estaban forjando. Y este es el objetivo de este artículo, acercarnos a ese valioso pensamiento pedagógico, que contribuyó, en un contexto muy plural de razas, lenguas y culturas que coexistían en un mismo territorio, al entendimiento entre ellas y a la construcción de un sistema educativo que podía ser compartido sin exclusiones de ningún grupo social. No es posible dar una respuesta de conjunto ahora a todos los matices que conlleva el estudio de esta influencia, tan solo se pretende destacar la percepción de la importancia que tenían para Harris la naturaleza y la educación artística en la formación de las personas, y establecer algunas comparaciones con las concepciones de Manuel B. Cossío en este ámbito.

\section{WILLIAM TORREY HARRIS COMO MODELO DE EDUCADOR EN LOS ESTADOS UNIDOS}

William Torrey Harris nació en una granja de Connecticut el 10 de septiembre de 1835, en plena naturaleza. Según Greenwood, ${ }^{11}$ su energía mental y su buena constitución física es la herencia que le dejó una infancia vivida al aire libre, en un ambiente de extremada pureza paisajística. Pasó por varias escuelas antes de llegar a Yale en 1854, de donde

\footnotetext{
10 «El movimiento de las ideas pedagógicas en los Estados Unidos», BILE, XXII (465), (1898): 362.

${ }^{11}$ Los datos biográficos de William T. Harris, cuando no se hace referencia explícita a otras fuentes, están recogidos de James M. Greenwood, Dr. William Torrey Harris, educator, philosopher and scholar: an address delivered before the Missouri StateTeachers' Association, at St. Louis, Mo., December, 28, 1909. [Sin datos de edición, copia en microficha conservada en la biblioteca del Ministerio de Educación, Cultura y Deporte].
} 
partió para Saint Louis en 1857, influenciado por las ideas de Theodore Parker (1810-1860). Allí empezó su labor como educador, bajo la dirección de Ira Divoll, en la Clay School, la primera escuela pública de la ciudad, en la que se atendía a jóvenes inmigrantes enseñándoles en su lengua nativa. ${ }^{12}$ Se preocupó de equipar su campo de juegos con aparatos para hacer ejercicios, y aunque mantuvo las enseñanzas clásicas de historia, gramática y matemáticas, incrementó el estudio de la botánica y la astronomía, llegando a construir y montar su propio telescopio. Divoll notó que aquel joven tenía mucho tacto y habilidad para tratar con los escolares y sus familias. Y enseguida destacó en una ciudad con una composición social muy diversa, desde antiguas familias españolas y francesas, hasta irlandeses y alemanes de más reciente asentamiento.

Sus formas afables y educadas, su discurso prudente y su riqueza de conocimiento, pronto le convirtieron en un favorito de todas las clases de la sociedad culta. Participó de forma sincera en los pensamientos y los sentimientos de sus nuevos amigos, interpretando acertadamente sus ideas y aspiraciones. En todos los sentidos era un hombre de escuela. Tenía una naturaleza muy receptiva, asimilaba muchos de los elementos de aquellas clases sociales que diferían de la del hogar de su infancia y se estuvo preparando para convertirse en un auténtico ciudadano de pleno derecho de los Estados Unidos y del mundo. ${ }^{13}$

En 1858 se creó la Asociación de Profesores del Estado de Misuri. Harris fue elegido secretario por consejo de Horace Mann, pero realmente el gran salto en sus percepciones pedagógicas se produciría cuando conoció ese mismo año a Henry C. Brockmeyer (1828-1906), quien llegó a los Estados Unidos en 1844 desde su Prusia natal. ${ }^{14}$ Harris estaba participando en un

${ }^{12}$ Carolyn Siemens Ward, Community Education and Crime Prevention. Confronting Foreground and Background Causes of Criminal Behavior (Westpor CT: Praeger, 1998): 214-215.

${ }^{13}$ Greenwood, Dr. William Torrey Harris, 8.

${ }^{14}$ Brockmeyer era un personaje fascinante, que en algunos aspectos recuerda a Henry Thoreau. Después de desempeñar varios empleos tras llegar de Europa con 16 años, entró como alumno en el Georgetown College de Kentucky, del que fue expulsado por cuestionar las ideas teológicas de su presidente. Trasladado a la Brown University tomó un contacto más fuerte con las ideas de Hegel, y al terminar sus estudios se fue a Warren County, en Misuri, donde vivió varios años en una cabaña, en plena naturaleza, en compañía de su perro y un fusil. Quería pensar libremente, pero deseaba también tener una granja, así que en 1858 se trasladó a Saint Louis buscando trabajo, encontrándose con Harris. 
debate en la Mercantile Library sobre filosofía oriental a la que asistió Brockmeyer, quien pensó que era la persona más sana de aquella reunión. Salieron juntos dando un paseo para seguir charlando, y Harris citó a Víctor Cousin, al que Brockmeyer rebatió. Fue el comienzo de su amistad y del grupo de estudiosos de la filosofía hegeliana que hubo en Saint Louis.

A Divoll, que había sido nombrado superintendente de las escuelas de Saint Louis en 1857, le admiró la discreción que mantuvo Harris durante la Guerra de Secesión, tratando que ningún fuego se avivase demasiado entre los escolares, lo que, acabado el conflicto, le granjeó el respeto de todos los alumnos y padres de familia, independientemente del bando en que habían luchado. Sin duda esos años se dio cuenta de la importancia de alentar cambios radicales en su país que procurasen una mayor igualdad entre las razas y grupos sociales, no ya porque la esclavitud estaba siendo erradicada de la cultura norteamericana, sino porque la sociedad tenía que organizarse de tal manera que los distintos grupos raciales y culturales pudiesen convivir y crecer juntos, una idea que hacía extensible al resto de países del mundo. Greenwood enfatizaba la importancia que tuvieron esos años, en que tomó contacto con las ideas de Hegel. Había reunido a su alrededor a un grupo de intelectuales que se preguntaba sobre lo que estaban pensando otros educadores en distintas partes del mundo, y mantenían vivos debates en el Club Filosófico que él mismo había fundado.

Cuando apareció en los Estados Unidos First Principles de Herbert Spencer, en 1862, su lectura le despertó tanto interés que le llevó a considerar que lo que sostenía Spencer era fundamental y a hacer un comentario critico entre sus amigos. A continuación envió su escrito a Atlantic Monthly, revista que entonces editaba James Thomas Field, pero fue rechazado para su publicación, y después se lo ofreció a la North American Review, con el mismo resultado. Fue el principio del Journal of Speculative Philosophy, la revista que editó entre 1867 y 1893, después de haber fundado en 1866, con Brockmeyer, la Saint Louis Philosophical Society, que le permitiría ponerse en contacto con algunos de los más importantes pensadores de otros países. En esta publicaron intelectuales de su entorno tan relevantes como William James, Stanley Hall o John Dewey. ${ }^{15}$ A este último lo conoció muy

${ }^{15}$ Como ya recordaba Gumersindo de Azcárate en 1879, haciéndose eco de un artículo de Stanley Hall, la revista era entonces el principal difusor del hegelianismo en los Estados Unidos y un importante nexo con la filosofía alemana, a lo que contribuía el intenso intercambio que se producía por los numerosos jóvenes que iban a estudiar a Alemania y los alemanes que iban a vivir a los Estados 
joven, y aunque mantuvieron después importantes divergencias intelectuales, a Dewey le seducía entonces la idea del organicismo de Harris tanto como el evolucionismo de Spencer, ${ }^{16}$ y ambos pensadores se encontraban en la base de sus formulaciones sobre la educación progresiva.

Ese mismo año en que empezó a editarse la revista fue nombrado asistente del superintendente de las escuelas de Saint Louis, cargo que todavía detentaba Divoll. Su debilitada salud le obligó a renunciar al año siguiente, y fue nombrado Harris para este puesto. El final de la Guerra de Secesión, en 1865, había producido migraciones internas muy notables de sudistas y unionistas. Los roces entre los nuevos y los viejos pobladores eran casi inevitables, y Harris fue consciente de que para construir el país no se podía hacer exclusión de ningún grupo. En 1868 había encargado un conjunto de informes sobre la situación de los niños de las zonas portuarias e industriales, y se encontró con que la mayoría dejaba de estar escolarizada antes de los diez años. Harris se propuso entonces saltarse el requisito de que no era necesaria la escolarización antes de los seis años. Estaba intentado ser comprendido cuando en 1871 regresó Susan E. Blow de Alemania, donde había conocido el funcionamiento del kindergarten. Después de varias conversaciones con Harris se trasladó a Nueva York para estudiar los métodos froebelianos con Maria Kraus-Boelté, ${ }^{17}$ regresando en mayo de 1873 a instancias de Harris. Así se abrió el primer kindergarten público de los Estados Unidos en septiembre de ese año, en Des Peres School, Carondelet, Missouri.

Harris fue además, durante esos años, valedor de la Escuela Normal de Kirksville que había fundado Joseph Baldwin en 1867. Entre 1870 y 1874 la visitaría dos veces al año, insuflándole su espíritu y modo de ver la educación y examinando los progresos de sus alumnos. ${ }^{18}$ Allí le conoció su biógrafo James M. Greenwood, quien recuerda que Harris era la

Unidos, a los que no solo ayudaba compartir el pensamiento protestante sino el espíritu del unitarismo que hacía más simple la aceptación de las verdades científicas desde posiciones religiosas. Véase «La filosofía de los Estados Unidos» BILE, III (51), (1879): 43.

${ }^{16}$ Lewis S. Feuer, «John Dewey and the Back to the People Movement in American Thought», Journal of the History of Ideas, 20 (4), (1959):568.

${ }^{17}$ Selwyn K. Troen, «Operation Headstart: The Beginnings of the Public School Kindergarten Movement», Missouri Historical Review, 66 (2), (1972): 213-218. Recuperado en http://statehistoricalsocietyofmissouri.org/cdm/compoundobject/collection/mhr/id/34014/show/33904.

${ }^{18}$ Sobre esta institución, véase E. M. Violette, History of the First District State Normal School Kirksville, Missouri (Kirksville: Journal Printing Company, 1905). 
figura central de un conjunto de educadores muy destacados entre los que menciona a G. C. Swallow, Louis Soldan, C. M. Woodward, Cook, Baldwin, Geo L. Osborne y Anna C. Brackett. Después de su primer viaje a Europa en $1880,{ }^{19}$ dejó su cargo de superintendente en Saint Louis y empezó a trabajar en la Concord School of Philosophy (Massachusetts), a la que financió y donde vivió retirado. Era una escuela filosófica para adultos que respondía a sus inquietudes, dirigida por Amos Bronson Alcott. Allí pudo desarrollar sus ideas en un ambiente que proseguía con los debates de la casa de Emerson. Allí se encontraba también Thomas Davidson, ${ }^{20}$ a quien Cossío había conocido en Roma en casa de Terencio Mamiani. ${ }^{21}$ Pero Bronson murió en 1888 y dos días después su hija, la

${ }^{19}$ Salió de Nueva York para Londres, el 14 de agosto de 1880 trasladándose de inmediato a Bruselas para participar en el Congreso Internacional de Enseñanza que organizaba la Ligue de l'Enseignement, pero no parece que en esta ocasión mantuviese ninguna conversación con Cossío. Véase K. F. Leidecker, Yanquee Teacher. The Life of William Torrey Harris (New York: The Philosophical Library, 1946), 390-393.

${ }^{20}$ Thomas Davidson (1840-1900). Su ideal de apeiroteísmo (el número de dioses que existen es infinito) influyó en William James y John Dewey. En 1880 había viajado durante ocho meses por Grecia a pie, cuestión que Cossío contaba con admiración cuando le conoció en Roma. Como consecuencia de este viaje escribió, con prólogo de Harris, The education of the Greek people and its influence on civilization (New York: Appleton and Company, 1895). Sobre la influencia en James y Dewey, véase Michael H. DeArmey, «Thomas Davidson's apeirotheism and its influence on William James and John Dewey», Journal of the History of Ideas, 48 (4), (1987): 691-707. Al igual que Harris, Davidson era un pensador muy preocupado por la inmortalidad y la existencia de un más allá tras la muerte.

${ }^{21}$ Davidson llegó a Boston en 1867 y enseguida se introdujo en el círculo de Amos Bronson Alcott. Su relación con el transcendentalismo es compleja. En aquellos años estaba hechizado con el positivismo de Comte, aunque, a su vez, interesado por la crítica alemana de la Biblia que comentaban los transcendentalistas, creía que el cristianismo debía ser purgado de su doctrinarismo rígido e instituciones. Entre 1875 y 1878 se trasladó a Cambridge donde fue tutor de los hijos de James Eliot Cabot, pero desilusionado con el protestantismo decidió viajar a Europa, y fue a Atenas para intentar comprender los fundamentos de la iglesia ortodoxa, que no le convencieron. Visitando las ruinas de la antigua Grecia tuvo una profunda experiencia religiosa que le llevaron desde una posición inicialmente panenteísta a considerar que la realidad consiste en un número infinito de sustancias espirituales. Cuando Cossío lo conoció en Roma, Davidson estaba experimentando esas transformaciones intelectuales que le llevaron incluso a debatir en una ocasión con el papa León XIII. Se sintió fascinado por los escritos de Antonio Rosmini Serbati (1797-1855), que estudió junto a los de Giordano Bruno, Leibniz y Kant, concluyendo que existía un "panpsiquismo monadológico» y que, si bien Dios existía en todas partes, existía completamente también en cada mónada. La realidad, decía, es Göttergemeinschaft, una sociedad de dioses. Davidson, aunque fue profesor y contertulio en la Concord School, no se sentía cómodo en su atmósfera que consideraba "demasiado etérea» y durante tres veranos, tras la muerte de Bronson Alcott, entre 1888 y 1890, creó su propia escuela en Farmington, Connecticut. Véase James A. Good «The Value of Thomas Davidson», Transactions of the Charles S. Peirce Society, 40 (2), (2004): 289-318. Véase http://www.autodidactproject.org/other/ TD.html (consultado el 18-VI-2016) con el título «The Development of Thomas Davidson's Religious and Social Thought». 
famosa escritora Louisa May Alcott, y enseguida el centro entró en decadencia. Harris era ya entonces, según Smith, uno de los doce educadores más influyentes de los Estados Unidos. ${ }^{22}$

Al regreso de su viaje a París en 1889, el presidente Benjamín Harrrison, a propuesta de Nicholas Murray Butler ${ }^{23}$, lo nombró superintendente del Buró Nacional de Educación el 12 de septiembre. ${ }^{24}$ Harris se instaló en la de noviembre y durante los diecisiete años siguientes dirigió la institución más importante de educación de los Estados Unidos, hasta el 30 de junio de 1906. ${ }^{25}$ Ejerció un fuerte liderazgo intelectual sobre los cambios y reformas que durante ese período se hicieron en la escuela y la educación secundaria. Era ingenioso y de convicciones profundas que nacían de su larga experiencia: presentaba sus puntos de vista con gracia y fuerza expresiva, al tiempo que mostraba una gran paciencia y generosidad de sentimientos hacia sus interlocutores. ${ }^{26}$

Unos rasgos que podrían recordar a Cossío, y que, como en su caso, tuvo que hacer frente a una fuerte oposición conservadora que no aceptaba un estilo tan personal y con tanta influencia entre el profesorado, hasta el punto que el año en que dimitió, James Hulme Canfiel señalaba que era un autor «citado con más frecuencia y con mayor aprobación por las revistas educativas y por los maestros de escuelas públicas que cualquier otro estadounidense, sin exceptuar a Horace Mann».27 Mowry

${ }^{22}$ Leonard G. Smith, A History of the United States Office of Education, 1867-1967 (Norman, Oklahoma: University of Oklahoma, 1967), 95. Tesis doctoral: https://shareok.org/handle/11244/2204 (consultado el 20-V-2016).

${ }^{23}$ Butler fue Premio Nobel de la Paz en 1931. Es la primera persona que propuso Harrison para el cargo de superintendente, pero renunció al mismo porque estaba enfrascado durante aquellos años en la creación del Teachers College de la Universidad de Columbia.

${ }^{24}$ El Buró de Educación de Washington había sido creado por una ley el 2 de marzo de 1867. Entre sus objetivos estaba unificar y hacer circular información entre todas las escuelas y centros de enseñanza de los Estados Unidos, así como poner las condiciones para el progreso de la educación y el mantenimiento de la eficiencia del sistema escolar. Sobre el nacimiento de este organismo, véase el folleto escrito por Alexander Shiras, The National Bureau of Education: Its history, Work, and Limitations, (Washington: Government Printing Office, 1875).

${ }^{25}$ Nadie volvería a ejercer ese cargo durante tanto tiempo. Harris falleció el 5 de noviembre de 1909.

${ }^{26}$ Smith, A History of the United States Office of Education, 1867 - 1967, 96.

${ }^{27}$ Smith, A History of the United States Office of Education, 1867 - 1967, 98-99. James Hulme Canfield (1847-1909) era presidente de la Ohio State University. Smith sugiere que, aunque Harris era un extraordinario intelectual, su gestión fue muy discutida, y que hacia 1900 su propia filosofía, en la que imbuía al Bureau, iba siendo relegada por el desarrollo que empezaban a alcanzar las ideas de John Dewey. 
se refería a él como el más abierto y tenaz educador que había producido los Estados Unidos. Poseía una gran capacidad de argumentación para convencer a sus contrarios, y recordaba que era un intelectual con muchos recursos: «Era un hábil y exitoso profesor, superintendente de escuelas, un escritor pulcro, un orador sencillo y convincente, expresando siempre pensamientos profundos, un correcto polemista y un astuto filósofo, quizá el mejor expositor de Hegel que ha tenido este país», decía rememorando su época de comisario. ${ }^{28}$ Cuando John R. Howard publicó Educational Nuggets y eligió a ocho educadores universales para explicar las tendencias de la nueva educación, terminó su antología con tres compatriotas: Harris, Butler y Charles William Eliot, ${ }^{29}$ probablemente los tres educadores más representativos de la pedagogía de los Estados Unidos a finales del siglo XIX.

\section{SOBRE EL PENSAMIENTO EDUCATIVO DE HARRIS}

Harris se planteaba las cuestiones fundamentales de la educación desde la perspectiva de la tensión entre la razón y los sentimientos. Un tema que examinó por el papel que desempeña la tragedia en comparación con la comedia en la educación, y que juzga con frecuencia mediante el valor escolar que se otorga a la literatura. Consideraba que

\footnotetext{
${ }^{28}$ William A. Mowry, «William T. Harris», The Journal of Education, 83 (4), (1916): 90-91.

${ }^{29}$ John R. Howard, Educational Nuggets: Plato, Rousseau, Herbart, Spencer, Harris, Butler, Eliot (New York: Fords, Howard \& Hulbert, 1899). Eliot, tenía unas ideas educativas que recuerdan a la ILE: defendía un progreso armonioso de los estudiantes desde el kindergarten hasta la universidad. Harris y Eliot formaron parte en 1892 del Committee of Ten, que promovió la National Education Association donde se manifestaron muchas de las contradicciones del sistema educativo norteamericano a finales del siglo XIX, presentes también en los países europeos, entre el peso que debían tener los programas clásicos y los experimentales en el curriculum. Harris, que defendía la importancia de las humanidades en la educación secundaria, se vio envuelto en una fuerte polémica con Charles De Garmo, presidente de la National Herbart Society, en la que salió derrotado. Véase Herbert M. Kliebard, The Struggle for American Curriculum, 1893-1958 (New York-London: Routledge Farmer, 2004, 3. ${ }^{\text {a }}$ ed.), 16-17. Los debates eran tan vivos que la National Education Association amplió el comité en febrero de 1893 a quince miembros (Committee of Fifteen) siendo nombrado Harris presidente del subcomité encargado de los programas de estudio en la educación elemental. Mientras que en el subcomité encargado de definir el programa de la formación de maestros hicieron un informe único firmado por todos sus componentes, y el de la organización de las escuelas urbanas tenía una única nota de discrepancia, el de la sección de Harris estaba firmado solo por él, presentando votos particulares a su propuesta el resto de los componentes. Véase Report of the Committee of Fifteen on Elementary Education with the Reports of subcommittees: on the training of teachers; on the correlation of studies in elementary education; on the organization of city school systems (New York-Cincinnati-Chicago: The American Book Company, 1895). La reacción de los herbartianos a las propuestas de Harris, según Kliebard, fue feroz.
} 
una persona podía estar en armonía o en discrepancia con la sociedad en que está inmersa. Cuando se vive en armonía con el medio social no se manifiestan los límites de la individualidad que solo aparecen cuando hay una colisión con el orden establecido. ${ }^{30}$ Mientras la comedia representa la derrota de los planes del individuo por sus propias contradicciones, la tragedia encarna el espíritu de innovación pero que todavía no es comprendido por sus coetáneos, y que a veces acaba en un gesto extraordinario que implica a la propia vida pero que deja una enseñanza, una conquista humana que perdura a lo largo de los siglos. Harris pone varios ejemplos deteniéndose especialmente en la muerte de Sócrates, pero también usó a El Quijote para explicar el abandono y deserción de los ideales clásicos del cristianismo y la adopción de otros nuevos tras el descubrimiento de América.

El niño comienza la vida «como salvaje que ignora la civilización» y hay que enseñárselo todo: "la manera de cuidar de su persona, la de conducirse ante los demás, la de hacer su obra en el mundo y ganarse la vida honradamente, la de observar y pensar» y es en la familia donde comienza a enseñársele a inhibirse. La escuela está, fundamentalmente, para educar la voluntad y el espíritu de la ciudadanía. Mientras en la familia el niño está "solo criado en el sentimiento del clan», la escuela ha de educarlo en la obediencia civil, ya que el maestro — dice- tiene «que contrarrestar la tendencia de sus discípulos a la conducta arbitraria y caprichosa; debe imponerles la forma de la razón en vez de su salvaje naturaleza, e insistir siempre en la adopción de formas prescritas». ${ }^{31}$ Harris está muy influenciado por Horace Mann cuando defiende la importancia de la escuela para extender el espíritu de civilidad. De hecho su estudio sobre Mann comienza analizando el paso de la ruralidad a la cultura urbana en los Estados Unidos, y termina destacando la importancia que daba a la educación de todos; de ilustrar a toda la población para apartarla de las tinieblas y mostrarles no solo el camino hacia la prosperidad y el honor sino también hacia la belleza. ${ }^{32}$

\footnotetext{
${ }^{30}$ William T. Harris, The educational value of the tragic as compared with comic in literature and art [Read before the Department of Superintendence, N. E. A., at Chattanooga, February, 1898. Sin otros datos de edición, copia en microficha conservada en la biblioteca del Ministerio de Educación, Cultura y Deporte].

${ }^{31}$ William T. Harris, «Los dos aspectos de la educación», BILE, XIX (422), (1895): 113.

${ }^{32}$ William T. Harris, Horace Mann (Syracuse, N. Y.: C. W. Bardeen, Publisher, 1896), 30-31.
} 
No es ajeno, por otra parte, al pensamiento transcendentalista norteamericano. Fue seguidor de las ideas de Ralph Waldo Emerson, y especialmente de su primer libro Nature, que había sido publicado en 1836. Así, antes de sumergirse en el idealismo alemán, había ya asumido los presupuestos los presupuestos de esta obra que aunaba naturaleza y arte, considerando que la primera era un escenario que se adecuaba por igual a la comedia y a la tragedia, cuyos placeres hay que usar con templanza "pues no siempre la naturaleza se disfraza con atavíos de fiesta, sino que el mismo escenario que ayer destilaba perfumes y relucía como para acoger la danza de las ninfas, extiende hoy su melancolía». ${ }^{33}$ En 1885, Harris publicó un estudio en el que señalaba que la poesía de Emerson abundaba en metáforas tomadas de las teorías modernas sobre la naturaleza. Admiraba sus poemas, que compara con «Under the Green Wood Tree» de Shakespeare. Creía que la belleza de la naturaleza exigía el abandono de las ideas de regularidad y simetría en el arte, ya que sugiere infinitos recursos en las formas, y por tanto nos hace libres.

Emerson sostenía que la naturaleza es el vehículo del pensamiento porque hacía consciente al hombre de «un alma universal», y que a través de ella llegábamos a la «unidad final» que contiene la verdad y la virtud que trasciende a la belleza. Harris destaca que Emerson encontraba en la naturaleza cuatro importantes beneficios: la resolución de necesidades básicas (alimento, vestido y vivienda), la belleza, el idioma y la disciplina. Centrándonos en la belleza, se detiene en que la belleza de la naturaleza presenta tres caras: el deleite, la señal de la virtud divina y el autoconocimiento, y que una obra de arte era la expresión de la naturaleza en miniatura que arroja luz sobre el misterio de la Humanidad. ${ }^{34}$

Es muy probable que su admiración por el pensamiento de Emerson facilitase su acercamiento al froebelianismo, en una síntesis de idealismo hegeliano y trascendentalismo, y que además esa conjunción hiciese más fácil la aproximación intelectual entre la pedagogía institucionista y el pensamiento de Harris, pues a pesar de las trazas diferenciales que separan al trascendentalismo del institucionismo — dice Pernas-, existen entre

\footnotetext{
${ }^{33}$ Ralph Waldo Emerson, Naturaleza (Palma de Mallorca: José J. de Olañeta, 2007), 30-32.

${ }^{34}$ William T. Harris, «Emerson's Philosophy of Nature», en The genius and character of Emerson. Lectures at the Concord Scholl of Philosophy, ed. F. B. Sanborn (Boston: James R. Osgood and Company, 1885), 346.
} 
uno y otro, "puntos de encuentro lo suficientemente significativos como para mirarlos conjuntamente». Uno de ellos es su aproximación al paisaje, como una dimensión que fragua la identidad y un referente moral que puede exorcizar la distancia entre lo culto y lo popular, y donde la naturaleza desempeña un papel fundamental para «regeneraciones identitarias», a veces en confrontación con tradiciones vernáculas muy arraigadas. ${ }^{35}$

Por otra parte, Harris, partidario de la escuela pública, fue quizá el primer educador norteamericano que ensayó reunir a blancos y negros en las mismas aulas. Decía que todos los niños, independientemente de su origen, debían compartir un mismo espacio escolar durante tres, cuatro o cinco años; que todos los segmentos de la sociedad debían tener experiencias comunes conviviendo en la escuela. No obstante, entendía que los individuos debían mantener el control de su propio destino, y que a la escuela correspondía cumplir el propósito fundamental de proporcionar a los niños disciplina mental, herramientas e inteligencia para ayudarse a sí mismos, pero que luego los individuos debían continuar su aprendizaje fuera de ella. Creía firmemente en educar a las personas en la idea de la autoayuda, e incluso defendía que un año de kindergarten podía ser crucial para el resto de la vida de una persona porque la exposición a sus juegos «la dotaba con la capacidad de captar exitosamente una amplia variedad de ocupaciones desde las labores de aguja al moldeado, a la preparación de la comida en el hogar». ${ }^{36}$

\section{ARTE Y NATURALEZA EN HARRIS}

Tal vez Cossío y Harris no se volvieron a ver después de aquel encuentro en París en 1889. Harris regresaría a Europa en 1895, acompañado por Greenwood, y visitó Alemania e Italia con detenimiento. Cossío no ha dejado tampoco constancia de que en su viaje a Estados Unidos en 1904 se entrevistasen. No obstante, compartían muchos ideales: eran dos educadores a los que les gustaba pensar en un mundo con mayores cotas de bienestar, inteligencia y humanidad, para lo que se necesitaba una labor educadora eficaz que llegase a toda la población. Ambos tienen concomitancias intelectuales

\footnotetext{
${ }^{35}$ Gonzalo Pernas, «De Concord a Guadarrama. Una semblanza compartida del trascendentalismo norteamericano y el institucionismo español», Revista de Occidente, 408 (2015): 91.

${ }^{36}$ Selwyn K. Troen, «Popular Education in Nineteenth Century St. Louis», History of Education Quarterly, 13 (1), (1973): 35-37.
} 
valiosas, a pesar de la lejanía geográfica y unas trayectorias vitales que se desarrollaron en circunstancias culturales muy diferentes.

Examinando los escritos de uno y otro podríamos encontrar muchos intereses comunes: difundir las bibliotecas escolares, la formación de los maestros, la escuela rural, la educación de párvulos y el kindergarten o la educación física. Pero si algo les asemeja intelectualmente es la importancia que concedieron al cultivo de las enseñanzas artísticas en la escuela, a saber despertar sentimientos estéticos en los niños y niñas, tanto con el arte como con la naturaleza. En los escritos de uno y otro podemos encontrar coincidencias, un universo intelectual compartido que se plasma en las realizaciones escolares de países alejados y que permanecían distantes. Hay tres textos de Harris con los que sin duda Cossío podría identificarse mucho: Beauty in Art vs. Beauty in Nature (s.a.), Art Education, the True Industrial Education (1889), y The Aesthetic Element in Education (1897). $\mathrm{El}$ arte, las relaciones entre arte y naturaleza, es probablemente uno de los temas que suscitaría un animado debate entre ambos, y queremos mostrar en este trabajo la importancia que estas cuestiones tenían para estos dos extraordinarios educadores.

Para Harris, al igual que para Cossío, el arte es un elemento fundamental en la educación de las personas porque está antes de cualquier manifestación intelectiva: atañe a los sentimientos, a las primeras manifestaciones anímicas que no precisan explicarse mediante la razón. Pero mientras Cossío lo apreciaba más desde la perspectiva de que permite gozar en su contemplación y creación, Harris lo apreció desde la perspectiva de la expresión de la libertad tanto en el cuerpo (arte griego) como desde el cuerpo (arte cristiano), o desde la inconsciencia del cuerpo y del alma (arte simbólico). La belleza en la naturaleza, se percibe — dice Harris— al salir de la ciudad: «Cuándo venimos al campo y estamos a solas con los árboles, las montañas, los prados, los arroyos y otros objetos naturales, tenemos una sensación de alivio frente a las obligaciones y a las preocupaciones que nos ocasiona nuestra red de relaciones» ${ }^{37}$ Pero, al mismo tiempo, sostiene que la naturaleza proporciona una sensación sedativa más que de belleza, porque la naturaleza no la apreciamos siempre por su belleza: es un reme-

\footnotetext{
${ }^{37}$ William T. Harris, Beauty in Art vs. Beauty in Nature, 4 [Sin datos de edición, copia en microficha conservada en la biblioteca del Ministerio de Educación]. El folleto no está fechado, aunque es posterior a 1889, ya que presenta a Harris como «U. S. Commissioner of Education».
} 
dio contra la presión de las obligaciones sociales y ofrece libertad, pero al mismo tiempo cree que podría establecerse una correspondencia simbólica entre el paisaje y el espíritu. Para desarrollar esta idea alude al ejemplo del paisaje pintado por Frederic Edwin Church, «Heart of the Andes», ${ }^{38} \mathrm{y}$, después de hacer una descripción del cuadro, señala:

Tal composición, con la diversidad en primer plano y las sublimes alturas de fondo bañadas por la luz del sol, ya es un símbolo de la naturaleza humana, la cual tiene pequeños detalles conformados por ocupaciones diarias, diversiones y decepciones, distracciones y dolor, esfuerzos y periodos de reposo, para nosotros mismos y para nuestros vecinos. Estas ocupaciones se encuentran en primer plano del cuadro de la vida humana. Del fondo surgen los elementos morales de nuestra naturaleza, la estructura de la razón en sí misma, sobre todo sus normas morales, su intuición de lo Divino, su fe religiosa y su perspectiva filosófica. Todos estos aspectos son abstractos como las nieves blancas en los glaciares, ningún verdor crece en ellos, pero, al mismo tiempo, abastecen los arroyos fertilizantes que descienden a las compuertas de la vida mortal. El arroyo de la vida y el arroyo de la bondad que vienen de lo Divino alegran la morada terrenal del hombre. ${ }^{39}$

Afirmaba que la correspondencia entre las cosas materiales y divinas es la base de la poesía y el arte, y que las personas sienten esta correspondencia en proporción al grado en que se posee una imaginación cultivada. ${ }^{40}$ Analiza con mucho cuidado el cuadro considerando que el arte simbólico

\footnotetext{
${ }^{38}$ Church pintó el cuadro en 1859, como resultado de una expedición a Ecuador en 1857. El lienzo, de grandes dimensiones, fue expuesto de inmediato en Nueva York provocando una gran conmoción pública para luego ser exhibido con el mismo éxito en Londres, donde Ruskin pudo admirarlo. Ese mismo año Darwin publicó Origin of Species, y falleció Alexander von Humboldt, a quien pocos años antes, en el segundo tomo de su obra Cosmos, había realizado un gran elogio de la pintura de paisaje. Véase Albert Ten Eyck Gardner, A. T. E.: «Scientific sources of the full-length Landscape: 1850», The Metropolitan Museum of Art Bulletin New Series, 4 (2), (1945): 59-65. http://www.jstor.org/ stable/3257164 (consultado el 14-II-2016).

${ }^{39}$ Harris, Beauty in Art vs. Beauty in Nature, 5.

${ }^{40}$ Harris solía poner ejemplos poéticos para expresar sus ideas. En este caso utilizaba el poema de Tennyson «The flower in the Crannied Wall» que permite saber, al arrancarla de la pared, todo lo que el hombre y Dios son. Consideraba que el hombre, como un ser inmortal, estaba en el peldaño más alto de la naturaleza y que la naturaleza, y que conectaba con el espíritu divino (The Educational Value of the Tragic, 3). Es una imagen poética que contrapone al cuadro de Church: la inmensidad del mundo material representado por las montañas que poco a poco se transforman en el verdor inago-
} 
no es el más alto grado de arte, sino el arte clásico cuando expresa una idea sagrada, porque la moralidad y la santidad son órdenes más elevados que la belleza. Pero al mismo tiempo — dice— ninguna religión es perfecta si condena la belleza, porque la belleza es buena siempre en sí misma.

La naturaleza es revelación de la divinidad —decía-, y sus leyes gobiernan el universo, pero no crea las instituciones humanas, no produce el arte ni las leyes que aseguran la justicia. La naturaleza no hace la escultura ni la literatura, que surgen en entornos civilizados muy evolucionados. Lo divino — afirma - no es la naturaleza salvaje inmediata, que es un escenario de violencia donde predomina más el destino que la libertad. Y el propósito divino culmina cuando el hombre surge en ella, inmortal, libre y responsable, a imagen de su creador. La naturaleza, aunque sea bella, no crea el arte porque el arte es libertad, y la naturaleza es bella por azar. De aquí, concluye, que la belleza del arte trasciende a la belleza de la naturaleza. ${ }^{41}$

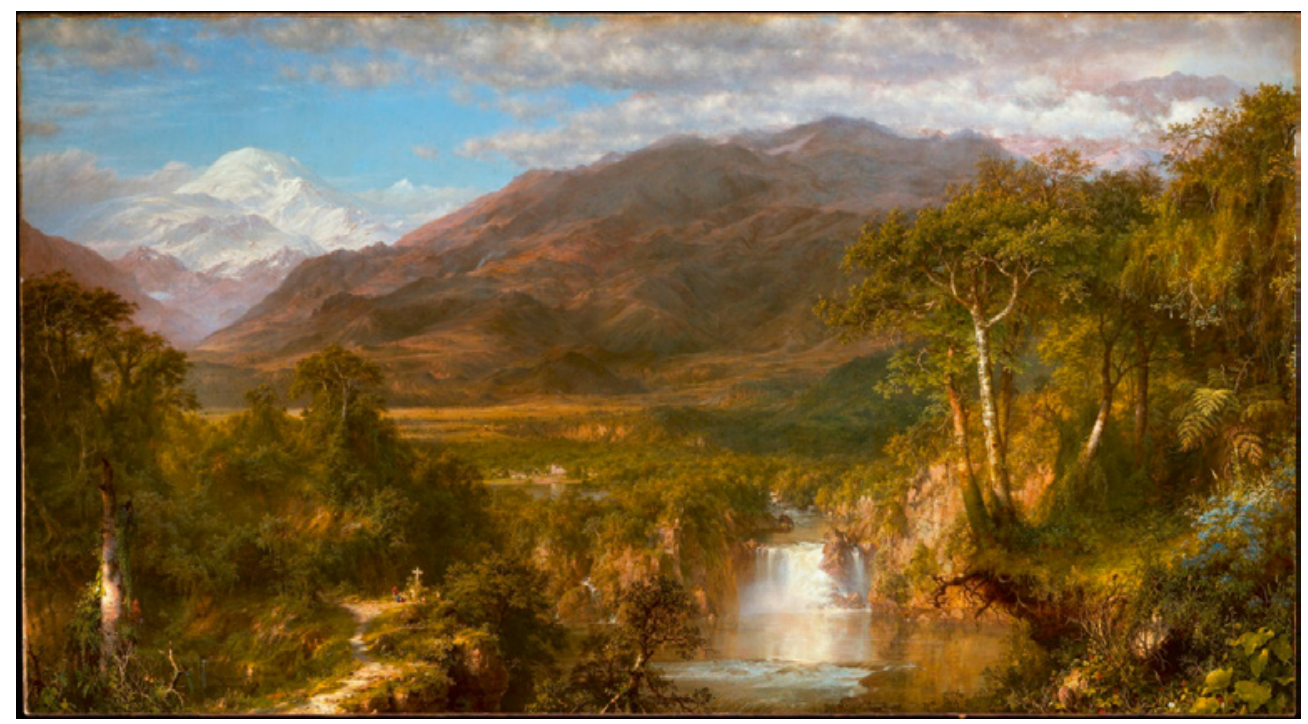

Frederic Edwin Church. Heart of the Andes. 1859. Metropolitan Museum of Art, Nueva York (http://www.metmuseum.org/collection/the-collection-online/search/10481)

table de la naturaleza, y aparece casi imperceptible la Humanidad y dentro de ella surge la excelsitud del cristianismo que da sentido completo al existir.

${ }^{41}$ Aunque sigue con bastante fidelidad la teoría de los tres estados del arte de Hegel, al contrario que el pensador alemán no considera el arte romántico (que engloba al arte cristiano) superior al clásico. 
Pocos días antes de venirse a Europa, en 1889, Harris había leído una memoria en la National Educational Association. Estaba todavía ligado a la Concord School, aunque en la publicación, hecha meses más tarde, figure ya como Comisario de Educación..$^{42}$ Es una disertación en la que se muestra, al igual que Giner y Cossío, muy preocupado con el engarce entre las enseñanzas técnico-profesionales y la formación general, ya que esta última la consideraba indispensable para la formación de la ciudadanía y una mejor capacitación de los trabajadores industriales. Harris defiende que una nación en la que todos sus trabajadores estén educados en el gusto por las formas bellas, este se reflejaría en las producciones, y redundaría en el bienestar del país y en un mayor y mejor comercio.

Para reforzar su argumentación recurrió a la formación artística que recibían los jóvenes suecos, recordando que Suecia era el país líder en la enseñanza del trabajo manual, así como a la labor que venía haciendo el Museo de South Kensington en Londres desde 1851. Harris establece una teoría de elaboración para las producciones industriales que resume en tres ideas: regularidad, simetría y armonía, que explica con amplitud. Claro está que para él la armonía es una cualidad superior que permite el acuerdo entre la voluntad y el cuerpo, entre la idea y su expresión. Después de hacer una revisión de las religiones griega, hindú, egipcia, asiria y persa y sus manifestaciones artísticas, concluye que el arte griego es primordial, porque explica la divinidad desde la propia corporeidad humana como expresión de la libertad personal.

Con todo, Harris no advertiría la importancia que tiene la enseñanza del arte como área de trabajo para la escuela común hasta 1897. En The Aesthetic Element in Education afirmó que hay cinco líneas de desarrollo intelectual que tienen una significación profunda en el aprendizaje escolar: matemáticas, gramática, el mundo orgánico y físico, la historia, y la formación estética, reuniendo en este último concepto la literatura y el arte. Ambas son manifestación de la energía de la mente, de la actividad propia de cada persona que hace perceptible el mundo interno a los sentidos. Pero se queja de que la escuela se ha ocupado siempre de la literatura, aunque no de otras artes, con la excepción de la música, y

\footnotetext{
${ }^{42}$ William T. Harris, Art Education. The True Industrial Education. A Cultivation of Aesthetic Taste of
} Universal Utility (Syracuse: NY, O. W. Bardeen Publisher, 1889). 
cree que la arquitectura, la escultura y la pintura podrían añadir «su sana lección» al currículum.

Partiendo de esta reflexión presentó un plan completo para desarrollar las bellas artes en la escuela, considerando que, en una escala ascendente, habría que hacer uso de cinco de ellas: arquitectura, escultura, pintura, música y poesía. Otras artes como la danza, la jardinería paisajística, el grabado, la elocuencia, el arte dramático o la oratoria, las consideraba derivaciones de esas cinco grandes artes. Su análisis es muy hegeliano: arte simbólico (Egipto), arte clásico (Grecia), arte romántico (Europa cristiana), aunque con matices en la importancia de los influjos que percibe en cada una de ellas. Si en la arquitectura y la escultura nada hay mejor que los logros alcanzados por los griegos, también creía que la pintura sublime es la del arte cristiano.

\section{LA FUNCIÓN DEL ARTE EN LA EDUCACIÓN PARA COSSÍO}

Cossío, en uno de sus escritos más conocidos sobre la enseñanza del arte, ${ }^{43}$ comienza aclarando que sus indicaciones solo están referidas a la arquitectura, escultura y pintura, lo que en comparación con las preocupaciones de Harris podríamos situarlo en la punta más avanzada de una novísima pedagogía. Lo importante de las reflexiones de Cossío es que, al igual que Harris, entiende muy bien que no hay «bellas artes y artes que no son bellas», en una clara inclinación a valorar las artes industriales y los objetos de uso fabricados por artesanos e industrias. En su caso concreto, en una clara referencia a las artes populares que en España tenían una fuerte impronta rural: - los cacharros de barro, el atuendo de los campesinos, los bordados y encajes, los aperos de labranza, la arquitectura rural- eran elementos sobre los que mostró siempre curiosidad, y ponía como ejemplo de la genialidad de los pueblos. Las cosas se hacen en principio porque sirven para algo, y algunas - decía Cossío- se hacen «simplemente para divertirnos, para que nos den placer, para que gocemos, porque al verlas nos gustan mucho

\footnotetext{
${ }^{43}$ Precedido por el antetítulo de «Conferencias normales sobre la enseñanza de párvulos», su trabajo «La enseñanza del arte» fue publicado en el BILE, IX (1885): 348-352 y X (1886): 57-62 y 100-101.
} 
y no sacamos de verlas más que ese gusto». Son las cosas «bonitas» o «bellas», que suele ser la idea de belleza más al alcance de un niño. ${ }^{44}$

Creía que la contemplación de obras de arte ejercitaba «el juicio crítico y el sentimiento de la belleza», incluso antes de que niños y niñas tuviesen edad para razonar, porque el arte permite que «amen lo bello, ennoblezcan sus gustos, gocen en los placeres más puros y sanos, y aprovechen, en suma, su cultura artística para vivir más refinada y bellamente». Pero para ello hay que poner al niño en contacto con los objetos, que sepa verlos, como ya manifestaba en 1879: «Enseñarle a pensar en todo lo que le rodea y a hacer activas sus facultades racionales es mostrarle el camino por donde se va al verdadero conocimiento, que sirve después para la vida». ${ }^{45}$ Pensaba que contemplar arte insuflaba "frescura y libertad de pensamiento» proporcionando elementos a la inteligencia para luego crear con las manos. Esta simplicidad del arte para despertar el pensamiento le condujo más adelante a que los campesinos conociesen las obras maestras de la pintura española a través del museo ambulante de las Misiones Pedagógicas ya que lo importante para Cossío era que avivase las conciencias, abriese luces a mundos que resultaban insólitos a quienes por primera vez los contemplaban, sin ninguna otra necesidad concreta o afán utilitario.

Cossío contaba con una gran habilidad para ponerse delante de un cuadro y hacer vibrar de entusiasmo a quienes le acompañaban. Al igual que Harris sabía sacar conclusiones de una pintura para entender la naturaleza y utilizaba el arte no ya para comprender la historia de un país, sino para entrar en las capas internas de lo que expresaba en todos los registros de las emociones, de descubrimientos que dejaban al aprendiz maravillado. Entre todas las descripciones que hizo sobre cuadros de El Greco hay una excepcional que nos sirve para contrastarla con el análisis que realiza Harris sobre la obra de Church. Me refiero al Entierro del Conde de Orgaz.

\footnotetext{
${ }^{44}$ Manuel B. Cossío, «La enseñanza del arte», en El maestro, la escuela y el material de enseñanza y otros escritos, ed. Eugenio Otero Urtaza (Madrid: Biblioteca Nueva, 2007), 165.

${ }^{45}$ Manuel B. Cossío, "Carácter de la pedagogía contemporánea. El arte de saber ver», en La Institución Libre de Enseñanza y Francisco Giner de los Ríos: Nuevas perspectivas. 3. Antología de textos (Madrid: Fundación Francisco Giner de los Ríos \& AC/E, 2013), 151.
} 


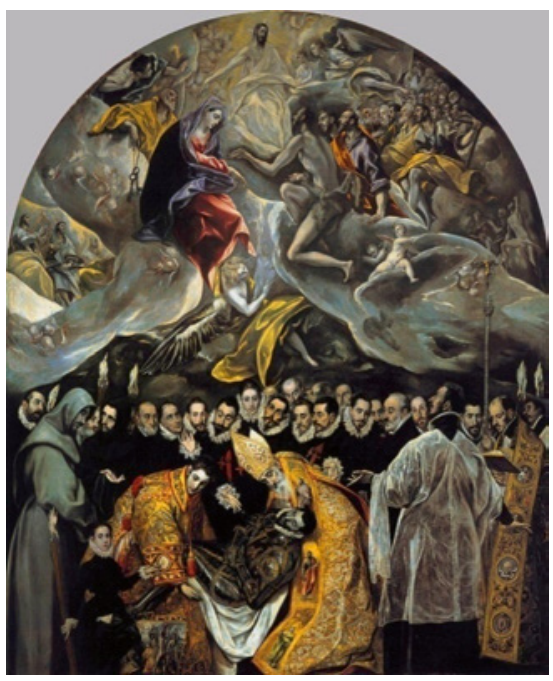

El Greco. El entierro del Conde de Orgaz. Iglesia de Santo Tomé (Toledo) 1587. (http://commons.wikimedia.org/wiki/File: El_Greco_-_The_Burial_of_the_Count_of_ Orgaz. JPG)

Mientras que en el cuadro de Church lo primero que vemos es la magnificencia de la naturaleza para luego acercarnos a los detalles de humanidad que se encierran en ella, en el cuadro de El Greco, vemos una región celestial arriba y la vida humana debajo, en su rostro cercano, viviendo la muerte con emoción y naturalidad, en una imagen también magnificente. En ambos hay una relación simbólica entre tierra y cielo, y sugieren que el tránsito por la vida se puede representar con instantes suspendidos que cumplen una función pedagógica: la inmensidad de la naturaleza y de la muerte, la poquedad humana ante una y otra, y ver el paso del tiempo entre un mundo celestial misterioso donde la fragilidad de la vida tiene la esperanza de la gloria eterna, y una naturaleza que lo inunda todo, y en la que la humanidad descubre también la grandiosidad de lo divino.

La descripción de Cossío es muy diferente a la de Harris. Compara El entierro con El Quijote, como obras cumbres de una cultura y de un tiempo que se recuerdan como memorables, pero la obra de El Greco - dice- destaca por la «honda intensidad contemplativa» que da a algunas de sus figuras "aire de enajenados». Un cuadro que propone una «mística a la vez naturalista y ascética», que representa una época de manera muy diferente a como lo hace Cervantes. Un espacio cerrado donde todo mira hacia adentro: «todo es esencialmente contemplativo; y cadáver, santos, monjes, clérigos y caballeros, todos parecen encerrados en su castillo interior, y en él deleitándose». ${ }^{46}$ Sin querer adentrarnos en una comparación que pudiese parecer inverosímil, interesa destacar que ambos educadores tenían una sensibilidad estética vigorosa, y que eran

\footnotetext{
${ }^{46}$ Manuel B. Cossío, "El Entierro del Conde de Orgaz», en La Institución Libre de Enseñanza y Francisco Giner de los Ríos: Nuevas perspectivas. 3. Antología de textos, 417.
} 
conscientes de que en la escuela había que desarrollar el gusto e interés hacia las obras de arte.

Además, Cossío concedía al arte un lugar central en la educación de una persona porque, afirmaba con radicalidad, que una vida que mereciese ser vivida tenía que transformarse en obra de arte. Decía que el origen del arte está en el juego y que los niños son artistas cuando juegan, y que la educación consistía en «enseñar al niño a ser artista de su propia vida, a reproducir su vida artísticamente». De ahí que concluyera que ser educado era el "arte de vivir bien», porque "saber vivir, es en sí mismo una obra de arte». El arte no estaba determinado por la necesidad, sino por el libre juego del espíritu, y apuntaba que la realidad se presentaba en tres dimensiones distintas: científica, práctica y creadora. Cuando esta última no tiene otra finalidad que el goce estético y desinteresado cuyo único producto es la belleza, nos encontramos en el meollo de la educación artística, eje de la educación de cualquier persona, porque defendía que un «alma embellecida» no podía realizar acciones toscas o brutales, y alcanzaba la verdad que es siempre bella. ${ }^{47}$

\section{DOS EDUCADORES QUE HICIERON PROGRESAR LA EDUCACIÓN}

A través del análisis comparado del pensamiento educativo de Cossío y Harris se ve cómo durante aquellos años el mundo occidental estaba influido por una pedagogía de cierto origen germánico , que había sido acogida con sucesivas transformaciones y adaptaciones locales en muchos países de Europa y América. En el fondo está la filosofía idealista -Krause en Cossío, Hegel en Harris- y más allá, la Naturphilosphie y el espíritu que representa Alexander von Humboldt; y, si se quiere, una pertinacia en buscar los valores educadores de la naturaleza siguiendo la estela que había abierto Rousseau. Arte y naturaleza, e incluso arte, ciencia y naturaleza no es solo una constante de la ILE, sino que sus ideas, que representa tan bien Cossío, deben enmarcarse en un movimiento trasnacional que comparte un ideario educativo que se caracteriza por el rechazo a las formas dogmáticas de organización de la existencia. Es la constatación de la superioridad del pensamiento científico sobre las

\footnotetext{
${ }^{47}$ Eugenio Otero Urtaza, Manuel Bartolomé Cossío: pensamiento pedagógico y acción educativa (Madrid: CIDE-MEC, 1994), 136-140.
} 
creencias religiosas, abriendo un nuevo horizonte de bienestar y optimismo, que además entendía que la naturaleza, como espacio de la ciencia y de la naciente cultura del medio ambiente, extendía su manto hacia el arte, el paisaje estético, la libertad y la imaginación creadora.

Esto es más perceptible si nos acercamos a las influencias anglosajonas. Tanto Harris como Cossío siguen una línea abierta por los prerrafaelistas ingleses, que continúa el movimiento Arts and Crafs fundado por William Morris, que reivindicaba el hacer con las manos frente a la creciente mecánica de la manufacturación que imponía la Revolución industrial. Y es aquí donde podemos ver algunas diferencias entre los dos educadores. Mientras Harris se muestra ilusionado por la perfección de un objeto que puede ser reproducido miles de veces, y de ahí su entusiasmo por los diseños industriales, Cossío admira las habilidades de los artesanos y sus obras únicas. Pero a ambos podemos considerarlos seguidores del pensamiento de Ruskin, porque asumían que el trabajo manual tenía un lugar central en la educación de los jóvenes, no ya porque desarrollaba habilidades mentales y creadoras, sino porque conducía a una persona a la obtención de muchas cualidades morales.

Aunque fueron defensores de la educación técnica y de que los jóvenes aprendiesen utilizando sus propias manos, entendían que la adquisición de habilidades y destrezas técnicas y el desarrollo de las artes industriales en sí mismas podría generar un caos si los esfuerzos educativos estaban mal dirigidos y faltaba la comprensión que producían esas acciones sobre nuestros sentimientos. Más allá de que fuese un motor de la creatividad en los escolares, la educación de la sensibilidad estética afectaba a la vida del alma. Cossío creía en el poder educativo de la naturaleza y a ella llevaba a los alumnos de la ILE todas las veces que podía. Pero su pasión íntima era la pintura, y especialmente los cuadros de El Greco. Veía en el misticismo que los envolvía la expresión de algo sublime que constituía la entraña del espíritu, un mundo interior en cada persona que difícilmente podría construirse con otro aprendizaje. En contraste, Harris se maravillaba con la naturaleza expresada en un lienzo que posiblemente veía como la expresión de la potencia inabordable de la divinidad, pero a su vez estaba extasiado con la pintura religiosa clásica, y, preocupado por la inmortalidad del alma. Tenía «hambre espiritual» y había dedica- 
do muchos esfuerzos a comprender el Juicio Final de Miguel Ángel o la Transfiguración de Rafael. ${ }^{48}$

No obstante, era Cossío quien preconizaba con más vehemencia que la escuela se instalase en un entorno de naturaleza. Harris defiende más la escuela como un instrumento que disciplina el espíritu indómito, que refrena las pasiones y entrena a las personas en la racionalidad y la sociabilidad, mientras que Cossío veía que a través del esfuerzo de una excursión al aire libre se desarrollaban valores personales, se afinaba el gusto estético y la curiosidad por conocer; y se fortalecía el carácter de una manera que no podía alcanzarse en las aulas. Sin duda ambos veían en el paisaje y en los elementos de la naturaleza una potencia educadora que atendía a todas las dimensiones de la persona y que, como el arte, inspiraba y formaba en la recreación del espíritu, y no olvidaba la existencia del cuerpo - tanto en su sentido clásico como cristiano y en sus ejemplos eximios que se mostraban en el arte-, de su cuidado y salud, y de la necesidad de abstraerse del trabajo mental con el recreo. Por ello salir a la naturaleza y disfrutar del aire, del sol y del paisaje era una tarea que no podía descuidarse por parte de maestros juiciosos. Pero era Cossío el que afirmaba que el campo escolar no tenía límites y que era fuera de la ciudad donde debía estar la escuela.

Mientras que para Harris el recreo era la intermisión del trabajo de los escolares que permitía el ejercicio físico y recuperar las fuerzas mentales, ${ }^{49}$ la idea de Cossío de escuela estaba más cerca de la pura recreación del espíritu. Además del «trabajo intelectual sobrio e intenso» para el que es necesario jugar y estar en contacto frecuente con la naturaleza y el arte, salir fuera amplia «la formación social que se atesora en el variar de impresiones, en el choque de caracteres, en la estrecha solidaridad de un libre y amigable convivir de maestros y alumnos» ${ }^{50}$ porque la excursión es la «verdadera escuela».

\footnotetext{
${ }^{48}$ C. H. Ames, «William Torrey Harris», The Journal of Philosophy, Psychology and Scientific Methods, VI (26), (1909): 708.

${ }^{49}$ William T. Harris, «Recess», Popular Education Document $n .{ }^{\circ} 20$. [Copia en microficha conservada en la biblioteca del Ministerio de Educación, Cultura y Deporte. Sin datos de edición, discurso leído en Washington el 13 de febrero de 1884].

${ }^{50}$ Manuel B. Cossío, «Principios pedagógicos de la Institución», en De su jornada (Madrid: Aguilar, 1966), 25.
} 
Nada hay en Harris que sugiera una camaradería andante entre profesores y discípulos, ni tuvo la afición por las excursiones a pie que se hacían en la ILE. La naturaleza era para él una referencia religiosa más que pedagógica. Un espacio que acercaba al hombre a la divinidad. Giner, más que Cossío, podía compartir esa pasión por los grandes espacios al aire libre, por el entusiasmo de sentarse a mirar el horizonte desde la cima de una montaña y pensar en la infinitud de las cosas, pero no tiene el fervor emersoniano de Harris. Cuando Giner escribió Paisaje en 1886, el mismo año que se creó la Sociedad para el Estudio del Guadarrama, hay en él un impulso muy complejo, más científico que metafísico y por supuesto con afán educador, quizá algo acentuado en la búsqueda de una identidad nacional española, pero no esencialmente místico. No excluye que el paisaje lleve al "recogimiento religioso», pero sin ir tan lejos como Harris y otros trascendentalistas que situaban ese momento de arrobamiento de la vivencia del paisaje natural como una puerta de comunicación con la divinidad: «la naturaleza es el órgano a través del cual el espíritu universal habla al individuo, y lucha por reconducir al individuo hacia él», afirmaba Emerson. ${ }^{51}$ Las pretensiones de Giner eran más sencillas, y por supuesto pedagógicas, como muestra su evocación de la puesta de sol desde los cerros de Guarramillas:

No recuerdo haber sentido nunca una impresión de recogimiento más profunda, más grande, más solemne, más verdaderamente religiosa. Y entonces, sobrecogidos de emoción, pensábamos todos en la masa enorme de nuestra gente urbana, condenada por la miseria, la cortedad y el exclusivismo de nuestra detestable educación nacional a carecer de esta clase de goces, de que, en su desgracia, hasta quizá murmura, como murmura el salvaje de nuestros refinamientos sociales; perdiendo de esta suerte el vivo estímulo con que favorecen la expansión de la fantasía, el ennoblecimiento de las emociones, la dilatación del horizonte intelectual, la dignidad de nuestros gustos y el amor a las cosas morales que brota siempre al contacto purificador de la naturaleza. ${ }^{52}$

\footnotetext{
${ }^{51}$ Emerson, Naturaleza, 104-105.

52 Francisco Giner de los Ríos, «Paisaje», en Por una senda clara, ed. de José García-Velasco y Eugenio Otero Urtaza (Sevilla: Centro Andaluz de las Letras, 2011), 43-44.
} 
No cabe duda que Cossío compartía este pensamiento de Giner, pero no tenía tanta pasión por la caminata como su maestro. Cuando Giner estaba tenso solía ir de paseo, mientras que Cossío prefería encerrarse en su habitación leyendo. Cossío admiraba los paisajes solemnes de la naturaleza, pero, al contrario de Harris, le gustaba el contacto con la ruralidad y admiraba la actividad de los campesinos. Veía en ella un tesoro decantado por siglos de generaciones e historia, e incluso un germen de transformación social y estilo de vida sano que contraponía al ruido y grosería de la ciudad. Nada de ello cabe encontrar en Harris cuyo apoyo a una escolarización formal pública estaba fundamentado en el desarrollo de las ciudades, en promover una ciudadanía productiva en una sociedad democrática.

Cossío buscó en el mundo rural un impulso para la modernización. Le interesaba más la naturaleza habitada. No como el fenómeno portentoso que Harris resalta en el cuadro de Church o el poema de Tennyson, sino por su admiración hacia comunidades humanas que habían sabido adaptarse con belleza en un entorno natural. Cossío era un gran admirador del arte popular cuyos productos «hunden siempre su firme raigambre en la entraña de la vida social, sin distinción de clases, y allí anidan y allí se perpetúan», y de cuyo fondo

brotan las diferenciaciones, las escuelas, los transportes de inspiración, los acentos de los genios creadores, y todo esto, nacido, al arte popular nuevamente revierte, y en él se incorpora, y él de ello se alimenta, como la madre tierra vive y se nutre a expensas de los seres que fecunda engendrara..$^{53}$

A pesar de algunas diferencias entre estos dos educadores, tan influyentes en su tiempo en sus propios países, comparten un conjunto de afinidades en referencia a la capacidad del arte y la naturaleza como potencias educadoras que merecen ser consideradas. Ambos forman parte del «transatlantic community of discourse», un complejo proceso de convergencia intelectual, más allá de los marcos nacionales de referencia, que buscaba una intermediación entre el idealismo y el positivismo, que

\footnotetext{
${ }^{53}$ Manuel B. Cossío, «Elogio del arte popular», De su jornada, 251-252.
} 
Kloppenberg llama la filosofía de vía media. ${ }^{54}$ Un debate que en España se mantuvo muy vivo en los primeros años de la Restauración tras el surgimiento del «krausopositivismo», pero que ciertamente no era un fenómeno doméstico, sino un ramal que se empezaba a extender entonces por toda la alta cultura occidental y que prosiguieron otros pensadores de las siguientes generaciones, con más aportaciones y nuevas perspectivas. Es incuestionable que Giner y Harris son de una misma generación y edad parecida, y que Cossío, más joven, forme parte de esa generación siguiente que se corresponde en Estados Unidos con la de John Dewey. Esto hace mucho más interesante examinar estos cruces generacionales, y acaso da pie a seguir buscando nuevas similitudes y diferencias en esta compleja trama de pensadores que han ido configurando los fundamentos de la educación contemporánea.

\section{Nota sobre el autor:}

Eugenio Otero Urtaza es licenciado en Filosofía y Letras (Sección de Pedagogía) por la Universidad Complutense de Madrid (1976) y doctor en Filosofía y Ciencias de la Educación por la Universidad de Oviedo (1990). Desde 1979 es profesor de la Universidad de Santiago de Compostela en el Campus de Lugo, y desde 1995 Catedrático de Escuela Universitaria de Teorías e Instituciones Contemporáneas de la Educación. Fue Director de la antigua Escuela de Formación del Profesorado de Lugo entre 1994 y 2003.

Destacado especialista en el estudio de la Institución Libre de Enseñanza, tema sobre el que ha publicado varios libros y más de setenta artículos y capítulos de libros, en los que ha abordado diferentes aspectos del florecimiento cultural y educativo desencadenado por Francisco Giner a partir de 1876. Se ha ocupado especialmente del estudio del Patronato de Misiones Pedagógicas, la trayectoria intelectual de Manuel B. Cossío, algunos de sus epistolarios inéditos, sus viajes e ideas pedagógicas.

Una buena parte de su esfuerzo investigador en los últimos años se centró en resaltar la dimensión internacional que tiene la Institución Libre de

\footnotetext{
54 James Kloppenberg, Uncertain Victory. Social Democracy and Progressivism in European and American Thought 1870-1920 (New York/Oxford: Oxford University Press, 1986), 10.
} 
Enseñanza, especialmente en sus relaciones con educadores alemanes, portugueses, belgas, franceses e ingleses. Es autor copartícipe en dos de los manuales del área más usados en España en la titulación de maestra/o de educación primaria e infantil (editados por Biblioteca Nueva y Graó) y actualmente coordina el grupo de investigación GI2124 de la USC (Edunartex) Educación, Naturaleza, Arte, Excursionismo. Desde 2011 coordina el máster en Dirección de Actividades Educativas en la Naturaleza. 